\title{
RESEARCH
}

\section{Changes in rates of upper gastrointestinal hemorrhage after the introduction of cyclooxygenase- 2 inhibitors in British Columbia and Ontario}

\author{
Muhammad Mamdani, Leanne Warren, Alex Kopp, J. Michael Paterson, Andreas Laupacis, Ken Bassett, \\ Geoffrey M. Anderson
}

\section{ABSTRACT}

Background: Population rates of upper gastrointestinal (GI) hemorrhage have been observed to increase with the introduction and rapid uptake of selective cyclooxygenase-2 (COX-2) inhibitors. Changes in COX-2 inhibitor use and upper GI bleeding rates in regions with relatively restrictive drug policies (e.g., British Columbia) have not been compared with changes in regions with relatively less restrictive drug policies (e.g., Ontario).

Methods: We collected administrative data for about 1.4 million people aged 66 years and older in British Columbia and Ontario for the period January 1996 to November 2002. We examined temporal changes in the prevalence of NSAID use and admissions to hospital because of upper GI hemorrhage in both provinces using cross-sectional time series analysis.

Results: During the period studied, the prevalence of NSAID use in British Columbia's population of older people increased by $25 \%$ (from $8.7 \%$ to $10.9 \% ; p<0.01$ ), as compared with a $51 \%$ increase in Ontario (from $10.9 \%$ to $16.5 \%$; $p<$ 0.01). Hospital admissions because of upper GI hemorrhage increased significantly in Ontario by about $16 \%$ on average, or about 2 admissions per 10 ooo elderly people, above expected values $(p<0.01)$. A similar increase was not observed in British Columbia.

Interpretation: More restrictive drug coverage policies, although limiting access to drugs and their potential benefits, may protect the population from adverse drug effects.

CMAJ 2006;175(12):1535-8

$\mathrm{T}$ he introduction of selective cyclooxygenase-2 (COX2) inhibitors, a group of NSAIDs, has resulted in a rapid increase in the number of people exposed to NSAIDs. Many patients who would otherwise not have used an NSAID are now using COX-2 inhibitors without a corresponding decrease in the use of more traditional nonselective NSAIDs. ${ }^{1,2}$ Although COX-2 inhibitors have been found to be associated with a lower risk of significant adverse gastrointestinal events than have traditional nonselective NSAIDs at the level of the individual patient, ${ }^{3,4}$ recent evi- dence suggests that the market expansion created by COX-2 inhibitors may increase rates of hospital admissions because of upper gastrointestinal (GI) bleeding at the population level.

The degree of adoption of a new drug into clinical practice is driven, in part, by decisions of public and private insurers to provide coverage. For example, $\mathrm{COX}-2$ inhibitors were listed in the Ontario Drug Benefit (ODB) formulary in April 2000 as limited-use drugs, which means that prescribers must indicate that the recipient has failed a trial of at least 3 nonselective NSAIDs or has clinically significant GI disease. In contrast, COX-2 inhibitors are highly restricted in British Columbia and can be obtained only through a "special authority" process, which involves a written request and approval by the drug plan. These differences in the nature and timing of reimbursement for COX-2 inhibitors have contributed to vastly different rates of NSAID use at the population level. ${ }^{6}$

To explore this issue further, we conducted a populationbased study to compare changes over time in the prevalence of NSAID use and rates of admission to hospital because of upper GI bleeding after the introduction of COX-2 inhibitors in the elderly populations of British Columbia and Ontario.

\section{Methods}

We conducted a population-based cross-sectional time series analysis using administrative health care databases from Ontario and British Columbia. We limited analyses to people 66 years of age and older in both provinces - a total of about I.4 million elderly people. The study period — January 1996 to November 2002 - was divided into 2I intervals of 120 days. The prevalence of NSAID use in each interval was determined by dividing the unique number of people dispensed any NSAID (either nonselective NSAIDs or COX-2 inhibitors) by the total number of people alive at the beginning of the interval. Similarly, we examined rates of admission to hospital because of upper GI hemorrhage (International Classification of Diseases, revision 9 [ICD-9] codes 53I, 532, 534, 578.o, 578.I and 578.9) obtained from the Canadian Institute for Heath Information Discharge Abstract Database, which contains a detailed record of all hospital admissions, including 
diagnostic and procedural information. Previous research has indicated that the ICD-9 codes have a positive predictive value of $86 \%$ in identifying upper GI hemorrhage.

Celecoxib was approved for use in Canada in April 1999. In April 2000, the ODB program listed both celecoxib and rofecoxib in its formulary as limited-use drugs for patients who have not responded to or were intolerant of traditional NSAIDs or patients with a history of upper GI hemorrhage or ulcer. In August 2000, PharmaCare, the public payer in British Columbia, listed the coxibs as "fourth-line" restricted drugs that require special authority approval by way of a formal request for funding.

Sources for prescription drug use were the ODB database in Ontario, which captures use of drugs reimbursed through the ODB program, and the PharmaNet database in British Columbia, which captures all prescriptions dispensed in the province, regardless of payer. The use of prescription drugs not reimbursed by the ODB in Ontario could not be captured. In British Columbia, dispensed prescriptions reimbursed by both private and public payers were captured.

Trends in potentially confounding medication use were also examined, with specific attention to prescription ASA, anticoagulants and antiplatelet drugs, proton-pump in- hibitors, histamine type- 2 receptor antagonists and selective serotonin re-uptake inhibitors. Time series analysis, ${ }^{8}$ involving autoregressive integrated moving average models and exponential smoothing models, was used to evaluate changes over time. Model-derived projections and their 95\% confidence intervals (CIs) for 6-month intervals from March 2000 through February 2002 were compared with actual utilization. Stationarity was assessed using the autocorrelation function and the augmented Dickey-Fuller test. ${ }^{7}$ The autocorrelation, partial autocorrelation and inverse autocorrelation functions were assessed for model parameter appropriateness and seasonality. The presence of white noise was assessed by examining the autocorrelations at various lags using the Ljung-Box $\chi^{2}$ statistic. ${ }^{7}$ Multivariate time series models were constructed to assess the impact of potentially confounding drug-use trends on the findings.

\section{Results}

The prevalence of NSAID use by British Columbia's elderly population increased from $8.7 \%$ just before the introduction of COX-2 inhibitors to $10.9 \%$ by the end of the observation period (Fig. I; $p<0.01$ ); this difference represents a $25 \%$ in-

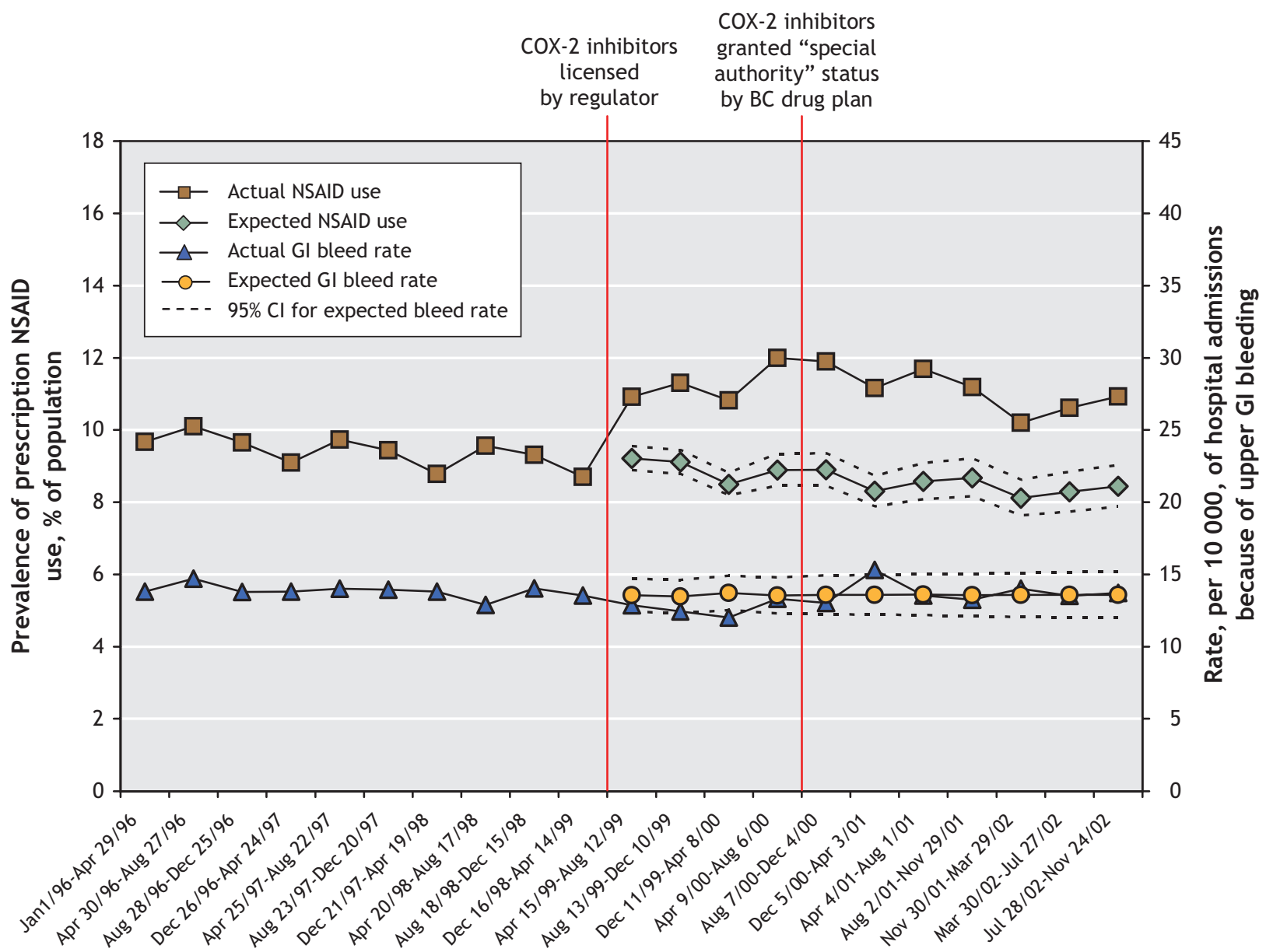

Fig. 1: Prescription use of NSAIDs by British Columbia residents aged $\geq 66$ years and rates of hospital admission because of upper gastrointestinal (GI) bleeding before and after listing of cyclooxygenase-2 (COX-2) inhibitors on provincial drug plan. 
crease in NSAID use and a relative increase of $29 \%$ over expected values. In contrast, NSAID prevalence in Ontario's elderly population increased from 10.9\% just before the introduction of COX-2 inhibitors to $16.5 \%$ by the end of the study period (Fig. $2 ; p<0.01$ ), for an increase of $51 \%$ and a relative increase of $64 \%$ over expected values. In both Ontario and British Columbia, the relative increases in NSAID prevalence were entirely attributable to new use of COX-2 inhibitors rather than to switching from nonselective NSAIDs to COX-2 inhibitors.

The rate of admission to hospital because of upper GI bleeding did not change significantly following the introduction of COX-2 inhibitors in British Columbia (Fig. I; $p=$ 0.62 ); instead, it remained stable at about 13.6 admissions per to ooo elderly people. The rate did increase significantly in Ontario, by about $26 \%$ above expected values on average, or about 2 admissions per to ooo elderly people (Fig. $2 ; p<$ o.or). The difference between the 2 provinces in rates of hospital admission because of upper GI bleeding as a function of the introduction of the COX-2 inhibitors was statistically significant $(p<0.0 I)$. Results from multivariate time series models accounting for temporal trends in potentially confounding drugs were similar to the original findings.

\section{Interpretation}

Although COX-2 inhibitors may be associated with a lower risk of upper GI bleeding than are traditional NSAIDs, they still increase this risk compared with no therapy. In this populationbased study, we observed significant differences in the amount of COX-2 inhibitor and overall NSAID use and in rates of hospital admission because of upper GI bleeding between 2 provinces in Canada that have different public drug-funding policies. The increase in hospital admission rates in Ontario following the introduction of COX-2 inhibitors is likely attributable to the province's relatively more liberal policy regarding drug plan coverage of COX-2 inhibitors. If COX-2 inhibitors do not simply replace the use of traditional NSAIDs but instead lead to marked market expansion, it is not surprising that their introduction leads to an increase in population rates of upper GI bleeding.

Several limitations of our study must be acknowledged. First, these data do not provide any information about the indication or appropriateness of use of NSAIDs, nor do they provide any information about the beneficial effects of these drugs. Therefore, the potential population harm must be weighed against the benefits of NSAID therapy when used appropriately. Second, this study was an ecological one, and although the problems of eco-

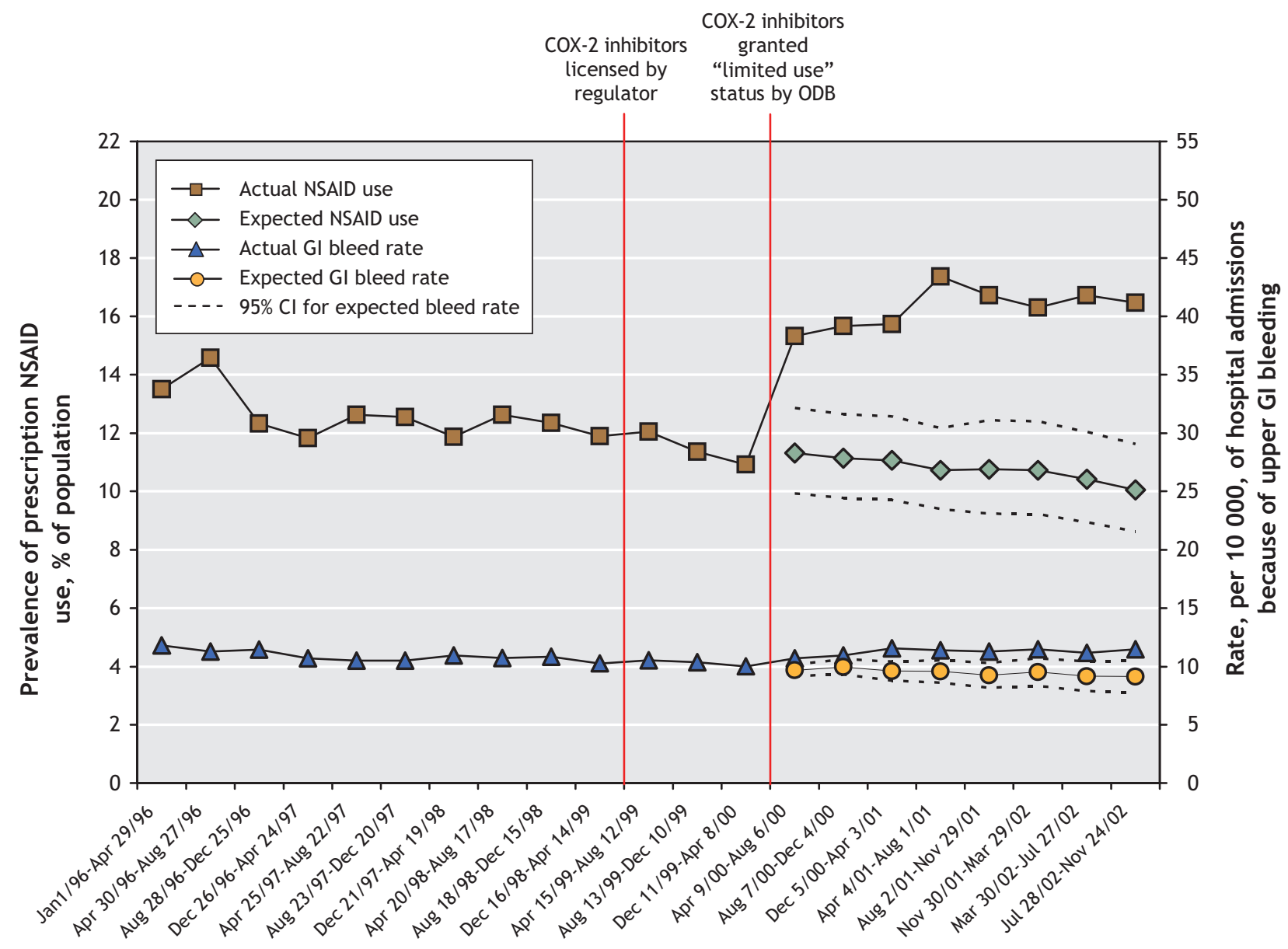

Fig. 2: Prescription use of NSAIDs by Ontario residents aged $\geq 66$ years and rates of hospital admission because of upper gastrointestinal (GI) bleeding before and after listing of cyclooxygenase-2 (COX-2) inhibitors on Ontario Drug Benefit (ODB) plan. 
logic fallacy are acknowledged, the intent was not to extrapolate ecologic effects to individual risks but rather to examine the system impact of the introduction of a drug group. Third, although problems with confounding are inherent in any observational analysis, we believe these to be unlikely given the close temporal association between the introduction of the COX-2 inhibitors and the increase in the rate of upper GI hemorrhage. Although we were able to assess changes in rates of potentially confounding use of drugs, such as proton-pump inhibitors and anticoagulants, we were unable to examine the use of nonprescription drugs such as acetaminophen, ASA and ibuprofen. The implications of not being able to capture this information are unknown.

Our findings suggest that population rates of upper GI hemorrhage following the introduction of COX-2 inhibitors differed between 2 provinces that have different reimbursement policies for these drugs. Restrictive formularies have been criticized for preventing appropriate access to potentially beneficial drug therapies. Our findings suggest that there may also be benefits to restrictive access.

\section{This article has been peer reviewed.}

From the Institute for Clinical Evaluative Sciences, Toronto, Ont. (Mamdani, Kopp, Paterson, Laupacis, Anderson); University of Toronto Faculties of Pharmacy (Mamdani) and Medicine (Mamdani, Laupacis, Anderson), Toronto, Ont.; Ministry of Health, Government of British Columbia, Victoria, BC (Warren); Therapeutics Initiative, the Department of Family Practice, the Centre for Health Services and Policy Research, and the Department of Pharmacology and Therapeutics, University of British Columbia, Vancouver, BC (Bassett) and the Sunnybrook Health Sciences Centre, Toronto, Ont. (Laupacis)

Competing interests: Muhammad Mamdani has conducted research in an unrelated content area at the request of an academic institution whose funding was supported by Pharmacia in the past 5 years, but none of the funding for this study was provided by any pharmaceutical company. He is currently an employee of Pfizer, Inc.

Contributors: Muhammad Mamdani, Michael Paterson, Andreas Laupacis, Ken Bassett and Geoffrey Anderson contributed to the conception and design of the study. Muhammad Mamdani, Leanne Warren and Alex Kopp contributed to the data acquisition and analysis. All of the authors contributed to the interpretation of the data. Muhammad Mamdani wrote the first draft of the manuscript, and the coauthors revised the manuscript for important intellectual content. All of the authors approved the final version to be published.
Acknowledgements: At the time of writing, Muhammad Mamdani was supported by a New Investigator Award from the New Emerging Teams (NETs) of the Canadian Institutes of Health Research (CIHR). Andreas Laupacis is a Senior Scientist of the CIHR. This study was supported by a CIHR operating grant (MOP-49527) and a CIHR Chronic Disease New Emerging Team Program grant (NET 54010). The NET program receives joint sponsorship from the Canadian Diabetes Association, the Kidney Foundation of Canada, the Heart and Stroke Foundation of Canada and the CIHR Institutes of Nutrition, Metabolism and Diabetes and of Circulatory and Respiratory Health.

The Institute for Clinical Evaluative Sciences (ICES) is a nonprofit research corporation sponsored by the Ontario Ministry of Health and LongTerm Care (OMHLC). The Centre for Health Services and Policy Research (CHSPR) and the Therapeutics Initiative (TI) are funded by the BC Ministry of Health (BCMOH). The opinions, results and conclusions expressed in this article are those of the authors; no endorsement by ICES, the OMHLC, CHSPR, TI or BCMOH is intended or should be inferred.

\section{REFERENCES}

I. Mamdani M, Rochon P, Laupacis A, et al. Initial patterns of use of COX-2 inhibitors by elderly patients in Ontario: findings and implications. CMAJ 2002;167 (I0):II25-6.

2. Girvin B, Rafferty T, Stevenson MR, et al. Uptake of COX-2 selective inhibitors and influence on NSAID prescribing in Northern Ireland. Pharmacoepidemiol Drug Saf 2004;13:153-7.

3. Mamdani M, Rochon P, Juurlink DN, et al. Observational study of upper gastrointestinal haemorrhage in elderly patients given selective cyclo-oxygenase-2 inhibitors or conventional non-steroidal anti-inflammatory drugs. $B M J$ 2002;325: 624-7.

4. Bombardier C, Laine L, Reicin A, et al. Comparison of upper gastrointestinal toxicity of rofecoxib and naproxen in patients with rheumatoid arthritis. $N$ Engl J Med 2000;343:1520-8.

5. Mamdani M, Juurlink DN, Kopp A, et al. Gastrointestinal bleeding after the introduction of COX 2 inhibitors: ecological study. BMJ 2004;328:1415-6.

6. Paterson M, Bassett K, Mamdani M, et al. What effects do provincial drug plan coverage policies for new drugs have on patterns of use and cost? Toronto: Institute for Clinical Evaluative Sciences; 2003. Available: www.ices.on.ca/file/ACFi2.pdf (accessed 2006 Oct 6).

7. Raiford DS, Gutthann SP, Rodriguez LAG. Positive predictive value of ICD.g codes in the identification of cases of complicated peptic ulcer disease in the Saskatchewan hospital automated database. Epidemiology 1996;7:I0I-4

8. Pindyck RS, Rubinfeld DL. Smoothing and extrapolation of time series. In: Econometric models and economic forecasts. 4th ed. New York: McGraw-Hill; I998. p. $467-88$.

Correspondence to: Dr. Geoffrey M. Anderson, Institute for Clinical Evaluative Sciences, Rm. GII6, 2075 Bayview Ave., Toronto ON $M_{4} N_{3} M_{5}$; fax 416 48o-6o48; geoff.anderson@utoronto.ca

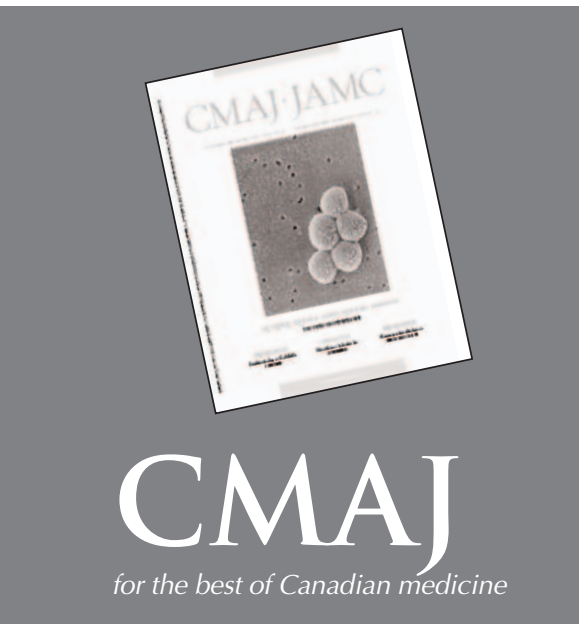

3000 volunteer reviewers from around the world are the foundation for CMAJ's thorough, criteriabased review process. The editorial staff includes scientific consultants with expertise in statistics, experimental design and epidemiology.

\section{CMA Member Service Centre}

tel $888855-2555$ or $613731-8610 \times 2307$

fax $613236-8864$

cmamsc@cma.ca

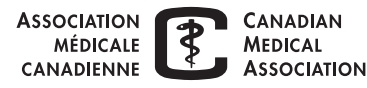

